\title{
HIGH TEMPERATURE ELECTRONIC STRUCTURE OF $\mathrm{Fe}_{3} \mathrm{O}_{4}$ *
}

Lu-San Pan and B. J. Evans

The University of Michigan, Ann Arbor, Mich1gan 48109

\section{ABSTRACI}

The high temperature electronic structure and conduction mechanism in $\mathrm{Fe}_{3} \mathrm{O}_{4}$ have been investigated through correlations of resistivity and magnetoresistance data with ${ }^{57} \mathrm{Fe}$ NGR. Similar to the resistivity, the relative $A$ and $B$ site isomer shifts are found to decrease rapidly at temperatures well below $\mathrm{T}_{\mathrm{N}}$. Above $\mathrm{T}_{\mathrm{N}}$ the $\mathrm{A}$ and $\mathrm{B}$ site patterns are unexpectedly poorly resolved and this is found to be due to a reduction in the difference in the electronic structure at the two sites. $\delta_{B}-\delta_{A}$ is only $0.22 \mathrm{~mm} \mathrm{~s}^{-1}$ at $855 \mathrm{~K}$ compared to the expected value of $0.32 \mathrm{~mm} \mathrm{~s}^{-1}$ assuming no change in the relative electronic structures at the two sites on passing through the magnetic order disorder transition. No significant changes in the ${ }^{57} \mathrm{Fe}$ NGR parameters are observed at the conductivity maximum near $400 \mathrm{~K}$. These results require a band model for the conduction mechanism above the Verwey transition and non-negligible conduction electron-ion core interactions for the A site ions above $\mathrm{T}_{\mathrm{N}}$.

\section{INTRODUCTION}

Desplte the many detailed studies of the Verwey transition in $\mathrm{Fe}_{3} \mathrm{O}_{4}, 1,2$ controversy still surrounds even a qualitative description of the electronic structure above or below the transition temperature, $\mathrm{T}_{\mathrm{V}}$. The transition has been described variously as a metal-insulator, semiconductor-semiconductor, and semimetal-semiconductor. Much of the recent attention has been devoted to phenomena at or near $\mathrm{T}_{\mathrm{V}}$. However, neither the transition nor the electronic structure of the low temperature phase can be adequately understood in the absence of a valid description of the high temperature phase. In addition, the electrical conductivity of $\mathrm{Fe}_{3} \mathrm{O}_{4}$ displays some remarkable variations above $T_{V}$ with a local maximum at $\leadsto 400 \mathrm{~K}$ and a minimum at $\sim 50 \mathrm{~K}$ below the Neel temperature, $\mathrm{T}_{\mathrm{N}} \cdot{ }^{3}$ These features of the electrical conductivity are poorly understood and have been subjected only recently to theoretical analysis. ${ }^{4}$

In the present investigation, ${ }^{57} \mathrm{Fe}$ nuclear gammaray resonance (NGR) measurements have been made on pure $\mathrm{Fe}_{3} \mathrm{O}_{4}$ from $77 \mathrm{~K}$ to $900 \mathrm{~K}$ to determine the thange (if any) in the electronic structure giving rise to the conductivity maximum at $\sim 400 \mathrm{~K}$ and the minimum just below $\mathrm{T}_{\mathrm{N}}$. By considering the broad systematics of the data, it was also hoped that a definitive conclustion could be reached concerning the high temperature electronic structure of $\mathrm{Fe}_{3} \mathrm{O}_{4}$.

Furthermore, because the ${ }^{57} \mathrm{Fe}$ NGR spectrum of $\mathrm{Fe}_{3} \mathrm{O}_{4}$ above $\mathrm{T}_{\mathrm{N}}$ is itself a conundrum, consisting of an apparent single line instead of two, partially resolved lines, a determination of the temperature at which the spectrum deviates from the expected behavior and a rigorous analysis of the line shape above $T_{N}$ have been made. From earlier measurements ${ }^{2}$ it was known that the ${ }^{57} \mathrm{Fe}$ NGR spectrum could be understood up to $750 \mathrm{~K}$ in terms of the $300 \mathrm{~K}$ spectrum; and consequent1y, the change in electronic structure giving rise to the apparent, single line above $T_{N}$ must take place within $100 \mathrm{~K}$ of $\mathrm{T}_{\mathrm{N}}$. This result suggests a possible relationship between the conductivity minimum below $T_{N}$ and the unusual character of the NGR spectrum above $\mathrm{T}_{\mathrm{N}}$. No ${ }^{57} \mathrm{Fe}$ NGR measurements have been specifically directed at determining possible changes in electronic structure associated with the conductivity maximum at $\sim 400 \mathrm{~K}$, and this temperature region has also received close attention in the present study. The results of this study demonstrate that there is indeed a relationship between the unusual character of the ${ }^{57} \mathrm{Fe}$ NGR spectrum above $\mathrm{T}_{\mathrm{N}}$ and the conductivity minimum below $\mathrm{T}_{\mathrm{N}}$. The change in electronic structure giving rise to both phenomena appears to be the onset of significant interactions between the $\mathrm{A}$ site $\mathrm{Fe}$ ions and the conduction electrons and the concomitant increase in spin-disorder scattering. The conductivity maximum at $\sim 400 \mathrm{~K}$ results in no noticeable anomalies in the NGR spectrum and is apparently due to higher order dynamical effects and not to fundamental changes in electronic structure, validating theoretical mode1s. ${ }^{4,5}$ The temperature dependence of the NGR parameters from $300 \mathrm{~K}$ to $750 \mathrm{~K}$ are consistent with band conduction and confirms recent theoretical predictions to this effect.

\section{EXPERIMENTAL}

The polycrystalline $\mathrm{Fe}_{3} \mathrm{O}_{4}$ sample has been described previously, investigated with a number of different techniques and is known to be highly stoichiometric. ${ }^{2}$

The NGR spectrometer consisted of an electromechanical velocity drive and a 1024 channel analyzer operated in the so-called "time mode". A $50 \mathrm{mCI}$ $\mathrm{Co}^{57} / \mathrm{Rh}$ source was employed and maintained at $298 \mathrm{~K}$ during the measurements. The spectrometer was calibrated with sodium nitroprusside and iron metal. A commercial design, vacuum furnace was used in the high temperature measurements, and the sample was contained in a boron nitride cup which was in contact with a chromel/alumel thermo-couple. The temperature was controlled with a proportional controller and was stable to within $\pm 2 \mathrm{~K}$ of the desired temperature.

\section{RESULTS}

The ${ }^{57} \mathrm{Fe}$ NGR spectrum of $\mathrm{Fe}_{3} \mathrm{O}_{4}$ at $856 \mathrm{~K}$ is shown in Fig. 1; $T_{N}$ for this sample is $855 \mathrm{~K}$. The solid line is the result of a least-mean-squares fit of a single, Lorentzian shaped line to the data. The line in the lower part of Fig. I, the "residual", is the difference between the fitted 1 ine and the experimental data; and the strong structure in the residual demonstrates the inadequacy of the single line fit. Even though the asymetry in the structure of the residual indicates the presence of unresolved structure in the NGR spectrum and not a simple deviation from Lorentzian shape as might result from instrumental and sample preparation effects, attempts to fit more than one line to the spectrum produced physically meaningless results in the absence of doubtful constraints during the fitting procedure.

The spectra below $800 \mathrm{~K}$ were straightforward and easily understood on the basis of the $300 \mathrm{~K}$ spectrum of $\mathrm{Fe}_{3} \mathrm{O}_{4}$. They were fitted to two, magnetic hyperfine + electric quadrupole patterns; the magnetic 

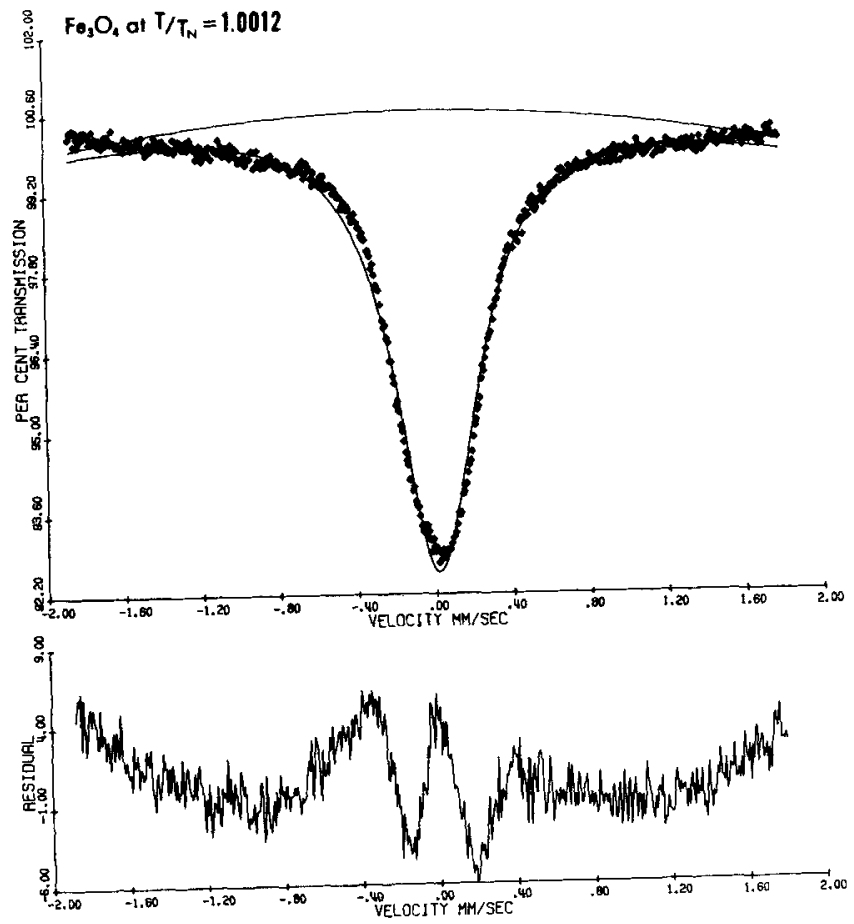

Fig. 1. ${ }^{57} \mathrm{Fe}$ NGR spectrum of $\mathrm{Fe}_{3} \mathrm{O}_{4}$ at $856 \mathrm{~K}\left(\mathrm{~T}_{\mathrm{N}}=\right.$ $855 \mathrm{~K})$. The points are the experimental data and the solid line is the least squares fit of a single, Lorentzian line to the spectrum. The lower line is the difference between the fitted spectrum and the experimental points. The deviations between $v-0.2 \mathrm{~mm} \mathrm{~s}-1$ and $v-0.6 \mathrm{~mm} \mathrm{~s} \mathrm{~s}^{-1}$, at the bottom of the absorption line, and between $0.1 \mathrm{~mm} \mathrm{~s} \mathrm{~s}^{-1}$ and $20.3 \mathrm{~mm} \mathrm{~s}^{-1}$ are to be noted and compared with Fig. 5. The relative heights of the minima in the residual are also to be compared with those in Fig. 5.

hyperfine field, $H(i)$, isomer shift, $\delta_{i}$, electric quadrupole interaction, $\Delta \mathrm{E}_{Q}(i)$, line-width, $\Gamma_{i}$, and intensities of each pattern were varied independently until a chi-square, goodness-of-fit criterion was met. The intensities and widths of lines 1,2 , and 3 in a given pattern were constrained to be equal to those lines 4,5 , and 6 of the same pattern.

None of the NGR parameters exhibited unusual behavior in the region of the conductivity maximum near $2400 \mathrm{~K}$. The difference in hyperfine flelds, $\mathrm{H}(\mathrm{B})-\mathrm{H}(\mathrm{A})$, increased from $30 \mathrm{kG}$ at $300 \mathrm{~K}$ to $45 \mathrm{kG}$ at $700 \mathrm{~K}$ in qualitative accord with the relative sublattice magnetisations. $\mathrm{H}(\mathrm{B})$ - $\mathrm{H}(\mathrm{A})$ decreased rapidly above $725 \mathrm{~K}$, but the significance of this decrease is complicated by the rapid fall in magnetisation in this temperature range and will not be considered further. The ratio of the integrated intensity of the $B$ site pattern to the A site pattern decreased from 1.90 at $300 \mathrm{~K}$ to 1.70 at $800 \mathrm{~K}$, exhibiting no anomalies and in accord with the recollless fractions of the two sites. $\Gamma_{B} / \Gamma_{A}$ is nearly constant from $300 \mathrm{~K}$ to $750 \mathrm{~K}$ but shows a substantial decrease above this temperature. Surprisingly, the decrease in $\Gamma_{B} / \Gamma_{A}$ is occasioned primarily by an increase in $\Gamma_{A}$. This increase in the $A$ site line-width is almost certainly related to the isomer shift anomaly to be discussed below.

In contrast to the behavior near $400 \mathrm{~K}, \delta_{A}$ exhibited an anomalous increase at $800 \mathrm{~K}$ as shown in
Fig. 2; and both $\delta_{A}$ and $\delta_{\mathrm{p}}$ decreased rapidly above this temperature. The difference in 1somer shifts, $\delta_{A}-\delta_{B}$, demonstrates more dramatically this change in isomer shifts as shown in Fig. 3.

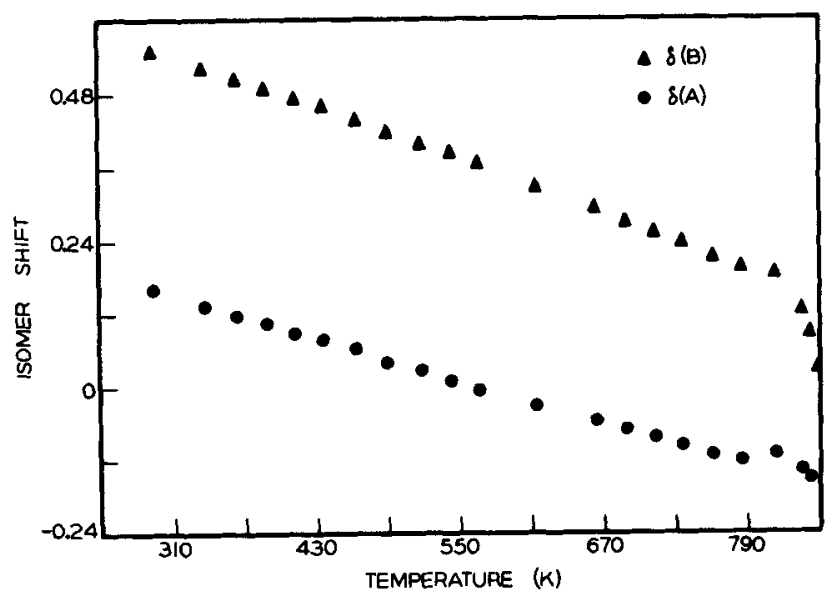

Fig. 2. The temperature dependences of the $A$ and $B$ site isomer shifts, $\delta(B)$ and $\delta(A)$, respectively. The isomer shifts are relative to a $\mathrm{Co}^{57} / \mathrm{Rh}$ source at $298 \mathrm{~K}$ and are uncorrected for the second-order-Doppler shift. The increase in $\delta(A)$ at $\sim 800 \mathrm{~K}$ and the rapid decrease in $\delta(B)$ above this temperature are noteworthy. The Neel temperature is offscale on the abscissa.

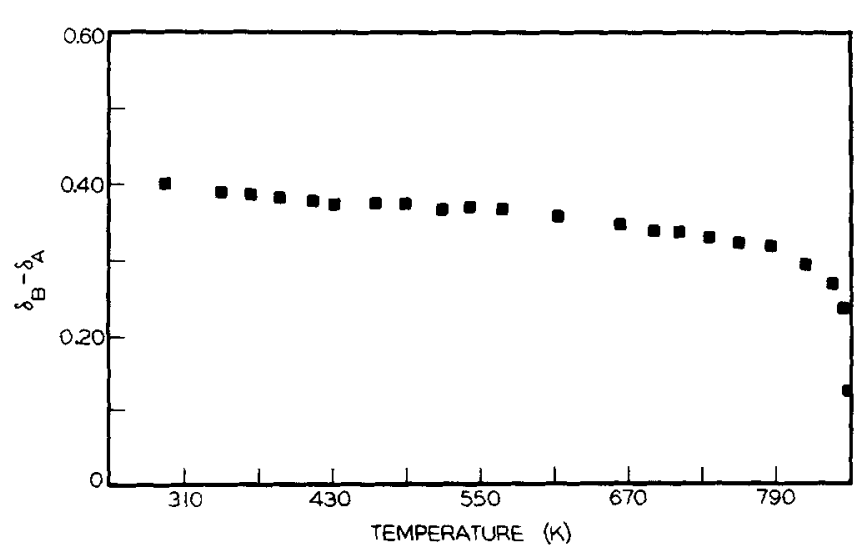

Fig. 3. The temperature dependence of the differential isomer shift at the $A$ and $B$ sites, $\delta_{A}-\delta_{B}$. The abrupt decrease above $\sim 800 \mathrm{~K}$ is clearly evident.

\section{DISCUSSION}

Insofar as the conductivity maximum near $400 \mathrm{~K}$ and the minimum approximately $50 \mathrm{~K}$ below $\mathrm{T}_{\mathrm{N}}$ are concerned, only $\delta_{i}$ and possibly $\Gamma_{1}$ provide insights as to thetr origins. None of the NGR parameters, including $\delta_{i}$, shows unusual behavior in the region of the conductivity maximum and it may be safely concluded that the maximum in the conductivity is not caused by a fundamental change or cessation of such change in the 
electronic structure. This result provides for the theoretical suggestions that the broad maximum in conductivity is due to dynamical scattering proceese. ${ }^{4}, 5$ The temperature dependence of $\delta_{1}$ near the high temperature conductivity minimum indicates that it is to be associated with a change in electronic structure. Below $750 \mathrm{~K} d \delta_{\mathrm{A}} / \mathrm{dT}$ and $\mathrm{d} \delta_{\mathrm{B}} / \mathrm{dT}$ have values of $-5.84 \times 10^{-4} \mathrm{~m} \mathrm{~s}^{-1} \mathrm{~K}^{-1}$ and $-7.34 \times 10^{-4} \mathrm{~mm} \mathrm{~s}^{-1} \mathrm{~K}^{-1}$, respectively. $\delta_{A}-\delta_{B}$ is $0.40 \mathrm{~mm} \mathrm{~s} \mathrm{~s}^{-1}$ at $298 \mathrm{~K}$ and on the basis of the above slopes of $\delta_{A}$ and $\delta_{B}$ below $750 \mathrm{~K}, \delta_{\mathrm{A}}-\delta_{\mathrm{B}} 18$ calculated to be $0.32 \mathrm{~mm} \mathrm{~s} \mathrm{~s}^{-1}$ at $855 \mathrm{~K}$. The NGR spectrum expected on this basis is shown in Fig. 4 and is quite unlike that in Fig. 1. We can now understand the poor resolution of the NGR

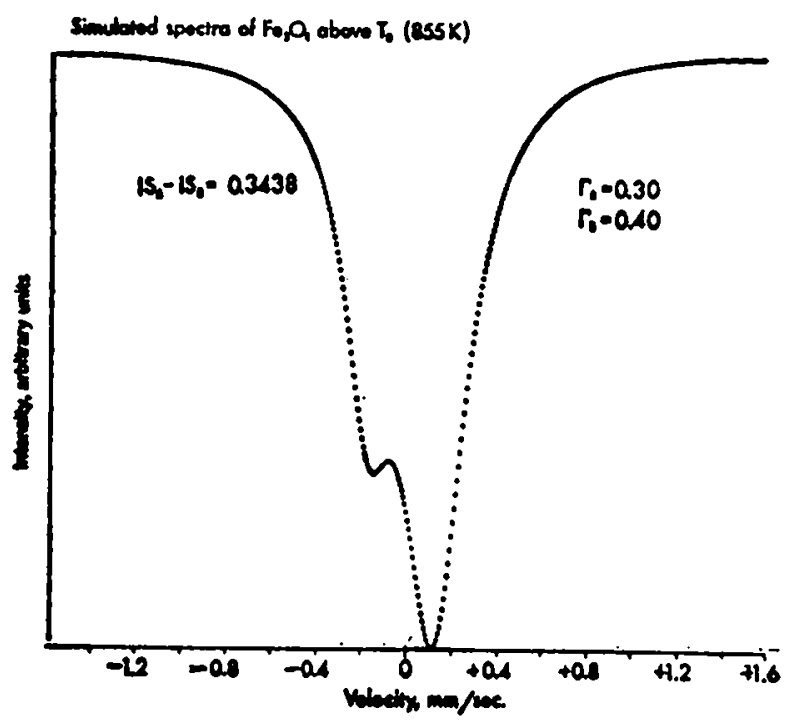

F1g. 4. Computed spectrum of $\mathrm{Fe}_{3} \mathrm{O}_{4}$ above the Neel temperature on the assumption that there are no changes in the electronic structure. The more intense Ine at more positive velocities is due to the $B$ site and the weaker, partially resolved Iine is due to the A site. The NGR parameters used to compute this spectrum are given in the figure.

spectrum of $\mathrm{Fe}_{3} \mathrm{O}_{4}$ above $\mathrm{T}_{\mathrm{N}}$ as being a consequence of a change in electronic structure at $2800 \mathrm{~K}$ as man1fested in the 1somer shifts shown in Figs. 2 and 3. The change in electronic structure is such that the s-1ike electron density at the A site increases relative to that at the $B$ site and approaches that of the $B$ site as $T_{N} 1 s$ approached.

Even though the spectra above $T_{N}$ cannot be fitted unambiguously, spectrum simulation permits a sem1quantitative estimate to be made of the 1somer shift difference. A large number of spectra were computed in which the $B$ to $A$ stie area ratio, $\Gamma_{B}$ and $\Gamma_{A}$ were assigned values derived from spectra below $T_{N}$ and $\delta_{A}-\delta_{B}$ was varied. These simulated spectra were then "fitted" with the ${ }^{57} \mathrm{Fe}$ NGR parameters obtained in the fit depicted in Fig. 1. The residual of such a spectrum in F1g. 5 corresponds most closely to the residual in Fig. I and indicates an 1somer shift difference, $\delta_{A}-\delta_{B}$, of $0.22 \mathrm{~mm} \mathrm{~s} \mathrm{~s}^{-1}$. Thus, the change in electronic structure comencing at $\sim 800 \mathrm{~K}$ results in a differential increase of $0.1 \mathrm{sm}^{-1}$ in
Ti Simulated spectra of fo, 9 above Th, (855K)
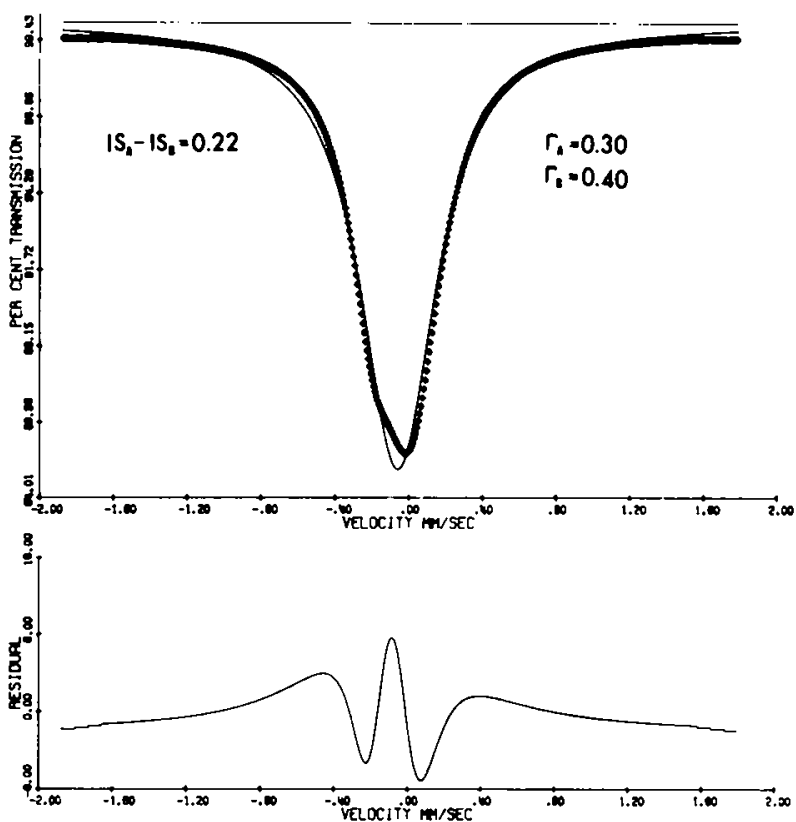

Fig. 5. Simulated spectrum of $\mathrm{Fe}_{3} \mathrm{O}_{4}$ above $\mathrm{T}_{\mathrm{N}}$. The points are the spectrum calculated assuming an isomer shift difference of $0.22 \mathrm{~mm} \mathrm{~s}^{-1}$ with all other parameters being the same as in Fig. 4. The solid line is the same as the line in Fig. 1 . The simllarities in the deviations of the line from the points, as indicated in Fig. 1, and the shape of the residual are remarkable.

$\delta_{A}$ relative to $\delta_{B}$ and indicates a slgnificant conduction electron - $\mathrm{Fe}^{3+}$ (A) ion core interactions.

An unambiguous mechanism for this interaction is difficult to come by but there are some plausible altermatives. At temperatures as high as $800 \mathrm{k}$, there is suffictent thermal energy to exclte electrons from the $B$ site band Into an $A$ site band and the rapid drop in magnetisation lessens the restrictions imposed by the antiparallel $A$ and $B$ site spin directions. It is also probable that a band overlap transition occurs between two, spin-split $A$ and $B$ site bands. Band overlap is permitted by the symetry of the $A$ and $B$ sublattices. E1ther of these mechanisms provides an unstrained explanation for the rapid decrease of $\delta_{\mathrm{A}}-\delta_{\mathrm{B}}$ as $\mathrm{T}_{\mathrm{N}}$ is approached.

The weak temperature dependence of $\delta_{1}$ and $\Gamma_{1}$ below $T_{N}$ and the absence of any incipient $\mathrm{Fe}^{2+}$ character in the NGR spectrum above $T_{N}$ makes a localized conduction mechanism for $\mathrm{Fe}_{3} \mathrm{O}_{4}$ above the Verwey transition unlikely. Thus the high temperature electronic structure of $\mathrm{Fe}_{3} \mathrm{O}_{4}$ is best described as that of a narrow-band, metallic or semimetallic, magnetic oxide. The unusual temperature dependence of the conductivity appears to be due to strong electron scattering resulting from temperature dependent magnetisations and electron phonon interactions.

\section{REFERENCES}

Support of this investigation by the National Sclence Foundation 1s gratefully acknowledged.

1. S. Chikazumi, Tech. Rept. ISSP A737, 1975.

2. B. J. Evans, AIP Conf. Proc. 24, 73 (1974).

3. R. Parker and C. J. Tinsley, Phys. Stat. Sol. a33, 189 (1976).

4. B. Lorenz and D. Ihle, Phys. Stat. Sol. b69, 451 (1975).

5. W. Haubenreisser, Phys. Stat. Sol. bly , 619 (1961). 